\title{
Snippets from the world wide web: A new feature
}

Sri Lanka Journal of Child Health, 2002; 31: 21-2

The World Wide Web / Internet is a wonderful storehouse of information for the discerning browser. Although some of the data in these electronic information system may be unsubstantiated, facts and figures, especially from reputed journals, make interesting reading and would be most useful to the judicious reader.

From this issue of our journal, a new feature, "Snippets from the World Wide Web", would be published. It would include selected catchy titles with a brief summary of the relevant information together with the address of the internet web site for direct access of the article.

We are grateful to Mary Reed Smith, WebMD/Medpulse of Medscape for giving us permission to reproduce their internet based material in this feature publication.

The Editors and the Editorial Board hope that this innovative feature will be helpful to our readers.

\section{Stella G de Silva \\ B J C Perera \\ Joint Editors}

Infertility therapy linked to birth defects and lowbirth-weight infants

The use of assisted reproductive technologies is associated with a significant increase in the risk of major birth defects and in the likelihood of having a low-birth-weight infant.

http://www.medscape.com/viewarticle/429648?src $\underline{\mathrm{mp}=\text { ped }-030802}$

\section{Nonsurgical management of infant clubfoot yields excellent results}

Using a series of casts to progressively modify pronation and internal rotation of the foot, orthopaedic surgeons are reporting excellent results with the nonsurgical correction of clubfoot in infants.

http://www.medscape.com/viewarticle/429202?src $\underline{\mathrm{mp}}=$ ped-030102

\author{
Small-for-gestational-age children may fare \\ poorly in school
}

Compared with their appropriate-for-gestationalage (AGA) counterparts, children who were smallfor-gestational-age (SGA) are more likely to show poor academic achievement in later years.

http://www.medscape.com/viewarticle/429372?src $\mathrm{mp}=$ ped -030802

Conservative measures not proven to relieve reflux in infants

Changes in infant positioning, changes in feeding, and other conservative measures do not have proven efficacy against gastroesophageal reflux disease in infants.

http://www.medscape.com/viewarticle/429037?src $\mathrm{mp}=$ ped-030102

\section{Wheezing in late childhood tied to adult asthma}

Although wheezing in early childhood often remits, such symptoms at the age of 11 years are associated with asthma in adulthood in individuals at high risk for atopy.

http://www.medscape.com/viewarticle/429299?src $\underline{\mathrm{mp}}=$ ped-030102

\section{Neurom uscular training reduces $A C L$ injury by $88 \%$ in female athletes}

Girls and women who play soccer, basket-ball, or volleyball are two to eight times more likely to injure the anterior cruciate ligament (ACL) than boys and men playing the same sports in the same positions.

http://www.medscape.com/viewarticle/428962?src $\underline{\mathrm{mp}}=$ ped -030102 
Fluticasone cream safe in children with atopic dermatitis

Few adverse systemic or skin effects were found when it was used for 4 weeks or less in children aged 3 months or older.

http://www.medscape.com/viewarticle/430508?src $\underline{\mathrm{mp}}=$ ped-032902

Circadian light cycle better than darkness for preterm infants

Cycled light promotes better weight gain than near darkness beginning at birth and at 32 weeks' postconceptional age.

http://www.medscape.com/viewarticle/430543?src $\mathrm{mp}=$ ped-032902

\section{Exclusive breastfeeding linked to improved} cognitive function in $S G A$ children

Children born small for gestational age (SGA) demonstrate improved cognitive function when exclusively breast-fed for the first 6 months of life.

http://www.medscape.com/viewarticle/430497?src $\mathrm{mp}=$ ped -032902

Low sodium intake in preterm infants linked to adverse neurologic outcomes

Premature infants who do not receive adequate amounts of sodium during the first 2 weeks of life demonstrate poor neurodevelopmental outcomes later in life.

http://www.medscape.com/viewarticle/430754?src $\underline{\mathrm{mp}=\text { ped }-032902}$ 